\title{
X-ray emission from hydrodynamical simulations in non-LTE wind models
}

\author{
J. Krtička ${ }^{1}$, A. Feldmeier ${ }^{2}$, L. M. Oskinova ${ }^{2}$, J. Kubát ${ }^{3}$, and W.-R. Hamann ${ }^{2}$ \\ 1 Ústav teoretické fyziky a astrofyziky, Masarykova univerzita, Kotlárská 2, 61137 Brno, Czech Republic \\ e-mail: krticka@physics.muni.cz \\ 2 Institut für Physik und Astronomie, Universität Potsdam, Karl-Liebknecht-Straße 24/25, 14476 Potsdam-Golm, Germany \\ 3 Astronomický ústav, Akademie věd České republiky, 25165 Ondřejov, Czech Republic
}

Received 5 June 2009 / Accepted 9 September 2009

\section{ABSTRACT}

\begin{abstract}
Hot stars are sources of X-ray emission originating in their winds. Although hydrodynamical simulations that are able to predict this X-ray emission are available, the inclusion of X-rays in stationary wind models is usually based on simplifying approximations. To improve this, we use results from time-dependent hydrodynamical simulations of the line-driven wind instability (seeded by the base perturbation) to derive the analytical approximation of X-ray emission in the stellar wind. We use this approximation in our non-LTE wind models and find that an improved inclusion of X-rays leads to a better agreement between model ionization fractions and those derived from observations. Furthermore, the slope of the $L_{X}-L$ relation is in better agreement with observations, however the X-ray luminosity is underestimated by a factor of three. We propose a possible solution for this discrepancy.
\end{abstract}

Key words. stars: winds, outflows - stars: mass-loss - stars: early-type - hydrodynamics - X-rays: stars

\section{Introduction}

Hot-star winds have been traditionally modelled assuming a spherically symmetric, stationary outflow. These assumptions provide a convenient basis for studying different phenomena that may influence the wind structure. The line profiles and wind parameters predicted in this way are in good agreement with those found from observations (e.g., Pauldrach et al. 2001; Vink et al. 2001; Krtička 2006).

However, a stationary approach to hot-star wind modelling was for a long time known to be not fully adequate (Lucy \& Solomon 1970). Hydrodynamical simulations show the growth of strong shocks in the wind due to the so-called line-driven instability inherent to radiative driving (Owocki et al. 1988; Feldmeier et al. 1997a). On the other hand, from the observational point of view the inhomogeneities do not imprint a clear signature in hot-star wind spectra in ultraviolet and visible spectral regions, and only a detailed spectral analysis reveals its possible presence (Bouret et al. 2003; Martins et al. 2005; Puls et al. 2006). Consequently, the influence of inhomogeneities (or clumping) on wind spectra has long been neglected. At present it is not clear whether the neglect of wind clumps in the formation of wind line profiles and infrared continua leads to an overestimate of mass-loss rates derived from observations (see Puls et al. 2008a, for a discussion of this problem).

The only clear observable signature of intrinsic wind nonstationarity is the existence of X-ray emission. This emission is directly observable for nearby stars (e.g., Berghöfer et al. 1997; Antokhin et al. 2008) and its existence can be inferred from the influence on the ultraviolet spectrum for those stars for which a direct observation of their X-ray emission is not available (e.g., Bianchi et al. 2004).

The line-driven instability is not the only possible production mechanism of X-rays in hot star winds. Because the winds are highly supersonic, any mechanism which causes wind stream collisions may also lead to X-ray production. Consequently, in hot star binaries the X-rays may originate due to the collisions of winds from individual stars (e.g., Prilutskii \& Usov 1976; Cooke et al. 1978; Pittard 2009). The collisions of individual wind streams channeled by the magnetic field may also cause X-ray emission (Babel \& Montmerle 1997; ud-Doula \& Owocki 2002). The former mechanism is important in binaries and the latter one in stars with a sufficiently strong magnetic field. In this paper we concentrate on a more general mechanism, operating in all radiatively driven stellar winds, i.e., the line-driven instability.

A central observational finding related to the X-ray emission is the dependence of the X-ray luminosity $L_{\mathrm{X}}$ on the total luminosity $L$ via the approximate relation $L_{X} \sim 10^{-7} L$ (e.g., Chlebowski et al. 1989). This relationship is still not explained by wind theory. Assuming a constant X-ray filling factor, Owocki \& Cohen (1999) showed that for stars with optically thick winds the observed X-ray luminosity scales with the massloss rate as $L_{\mathrm{X}} \sim \dot{M}$, and for stars with optically thin winds as $L_{\mathrm{X}} \sim \dot{M}^{2}$. As the predicted wind mass-loss rate scales with the stellar luminosity as $\dot{M} \sim L^{1 / \alpha^{\prime}}$ (Kudritzki \& Puls 2000), where $\alpha^{\prime} \approx 0.6$ for luminuous $\mathrm{O}$ stars $^{1}$ (e.g., Vink et al. 2000; Krtička \& Kubát 2009; Puls et al. 2008b), the predicted slope of the $L_{X}-L$ relation is steeper than the observed one. This difference may originate in the radial dependence of the X-ray filling factor (Owocki \& Cohen 1999) or may be caused by the dependence of the cooling length on the wind density (Krtička \& Kubát 2009). Note also that macroclumping (Feldmeier et al. 2003; Oskinova et al. 2004; Owocki et al. 2004; Oskinova et al. 2006) may lead to a decrease in the effective opacity in the X-ray region and affect the X-ray luminosity.

Although a real hot-star wind should be far from stationary and spherically symmetric (on small scales), the success of

\footnotetext{
1 Note that the situation may be different in late $\mathrm{O}$ and $\mathrm{B}$ supergiants, for which the value $\alpha^{\prime} \approx 0.8-1$ might be a more appropriate one (Benaglia et al. 2007).
} 
wind modelling applying these approximations motivates us to incorporate the inhomogeneities found in time-dependent hydrodynamical simulations into a spherically symmetric, stationary wind model. These models can serve as an efficient tool to study hot-star winds until more elaborate hydrodynamical simulations that consistently take into account the non-LTE effects become available.

There have been earlier attempts to include X-ray emission in non-LTE wind models. They were either based on simplified analytical models (Feldmeier et al. 1997b; Martins et al. 2005), or the X-ray emission was included using ad hoc free parameters (aka the "filling factor", e.g., MacFarlane et al. 1994; Martins et al. 2005; Krtička \& Kubát 2009) describing the hot wind part. Here we use the results of available hydrodynamical simulations of Feldmeier et al. (1997a) to directly describe the X-ray emission in a compact form and include it in our non-LTE wind models.

\section{Hydrodynamical simulations}

The hydrodynamical simulations we employ here to estimate the $\mathrm{X}$-ray emission from $\mathrm{O}$ stars were performed using the smooth source function method (SSF, Owocki 1991; Owocki \& Puls 1996). This corresponds roughly to a formal integral of the radiation force using a pre-specified line source function, here the optically thin source function, $S \sim r^{-2}$. The line-driven instability that is responsible for the formation of X-ray emitting shocks in the wind is captured by a careful integration of the line-optical depth with a resolution of three observer-frame frequency points per line Doppler width and accounting for non-local couplings in the non-monotonic velocity law of the wind (see Owocki et al. 1988 , for details). The angle integration in the radiative flux is limited to one single ray which, however, is not the radial ray but hits the star at $\approx 0.7 R_{*}$. This accounts with sufficient accuracy for the finite-disk correction factor (Pauldrach et al. 1986) and avoids the critical-point degeneracy of the point-star CAK model (Poe et al. 1990).

To avoid any artificial overestimate of the X-ray production in the wind, special care was taken in the numerical calculation of the line force on the staggered spatial mesh in order to correctly reproduce the line-drag effect (Lucy 1984), which is responsible for a partial reduction of the instability, especially in layers close to the star (Owocki \& Rybicki 1985). The pure hydrodynamics part of the code is a standard van Leer solver following standard prescriptions for such a scheme: staggered mesh; operator splitting of advection and source terms; advection terms in a conservative form using van Leer's (1977) monotonic derivative as an optimised compromise between stability and accuracy; Richtmyer artificial viscosity; and non-reflecting boundary conditions (Hedstrom 1979; Thompson 1987, 1990). The numerical collapse of post-shock radiative cooling zones caused by the strong oscillatory thermal instability (Langer et al. 1981 ) is prevented by artificially modifying the radiative cooling function below a certain temperature at which X-ray emissivity is still small. Further details can be found in Feldmeier (1995).

As seed perturbations for unstable growth we introduce a turbulent variation of the velocity at the wind base, at a level of roughly one third of the sound speed. This perturbation is obtained from a simple quadrature of the Langevin equation (see Risken 1996) with a coherence time in the friction term of $5000 \mathrm{~s}$, which is well below but not too far off the acoustic cutoff period of the star at which, according to the theorem by Poincare (see Lamb 1945), acoustic perturbations of the photosphere should accumulate. The power spectrum $E(k)$ of this
Langevin turbulence has a spectral index of -2 , which is close to the Kolmogorov index of $-5 / 3$ for the universal, inertia subrange of turbulence.

The variety of dynamical structures in the instabilityinduced, line-driven wind turbulence (which grows out of but is not identical to the turbulence applied in the photosphere via boundary conditions) is still largely unexplored, but seems to be similarly intricate to that found in other turbulent flows in ordinary fluids and gases or in astrophysical MHD settings. There are indications of the presence of a quasi-continuous hierarchy of density and velocity structures in the wind, similar to that found in supersonic Burgers turbulence with shock cannibalism (Burgers 1974), and there are also indications of a separation of dense structures into two distinct families, which we address under the names "shells" and "clouds". The shells are formed close to the star, in a first stage of unstable growth. They do not collect all the wind material but rather one half of it, since the negativevelocity perturbations remain unaffected by the line-driven instability (MacGregor et al. 1979). Further out in the wind, in a second stage of unstable growth, clouds are "ablated" from the outer rim of the remaining mass reservoir at CAK densities, and are accelerated by the stellar radiation field through the emptied regions, eventually colliding with the next-outer shell, and producing observable X-ray flashes in this collision (Feldmeier et al. 1997a).

Without photospheric Langevin turbulence, clouds do not occur and the mass reservoir is continuously fed into the next outer shell via a thin stream of fast gas that by far cannot (see Hillier et al. 1993) account for the observed X-ray emission from $\mathrm{O}$ stars. To test for the relevance of the specific form of photospheric turbulence, we also calculated models with a "stochastic" photospheric sound wave as seed perturbation for the instability, i.e. a sound wave with stochastic variations in period, amplitude, and coherence time, which resulted in largely the same results.

\section{X-ray emission from hydrodynamical simulations}

To incorporate the results of hydrodynamical simulations of $\zeta$ Ori A wind in non-LTE wind code in a manageable way, we approximate the emission from hydrodynamical simulations as a polynomial function. This could be done in two ways. The first way is to calculate the X-ray emission based on a local values of hydrodynamical variables, and then determine a function that best approximates its radius and frequency dependence (see Sect. 3.1). The second, somewhat simpler way, is to find a polynomial that fits the temperature structure of the simulation, and then use this polynomial to calculate the emission (see Sect. 3.2).

\subsection{Spectrum fitting}

First, the wind structure from hydrodynamical simulations is used to calculate the X-ray emissivity as a function of radius and frequency. We divide the wind model into 29 equally spaced radial bins and calculate the total wind X-ray emission in each radial bin at the timestep $k$ as an integral

$l_{i j k}=\int_{r_{i}}^{r_{i+1}} r^{2} n_{\mathrm{e}}(r) n_{\mathrm{H}}(r) \Lambda\left(v_{j}, T(r)\right) \mathrm{d} r$

where the radius $r_{i}$ denotes the radial boundary of the $i$ th bin, $n_{\mathrm{e}}(r), n_{\mathrm{H}}(r)$ are the number densities of free electrons and hydrogen, and the X-ray emissivity $\Lambda\left(v_{j}, T(r)\right)$ is calculated using the Raymond-Smith X-ray spectral code (Raymond \& Smith 1977; 

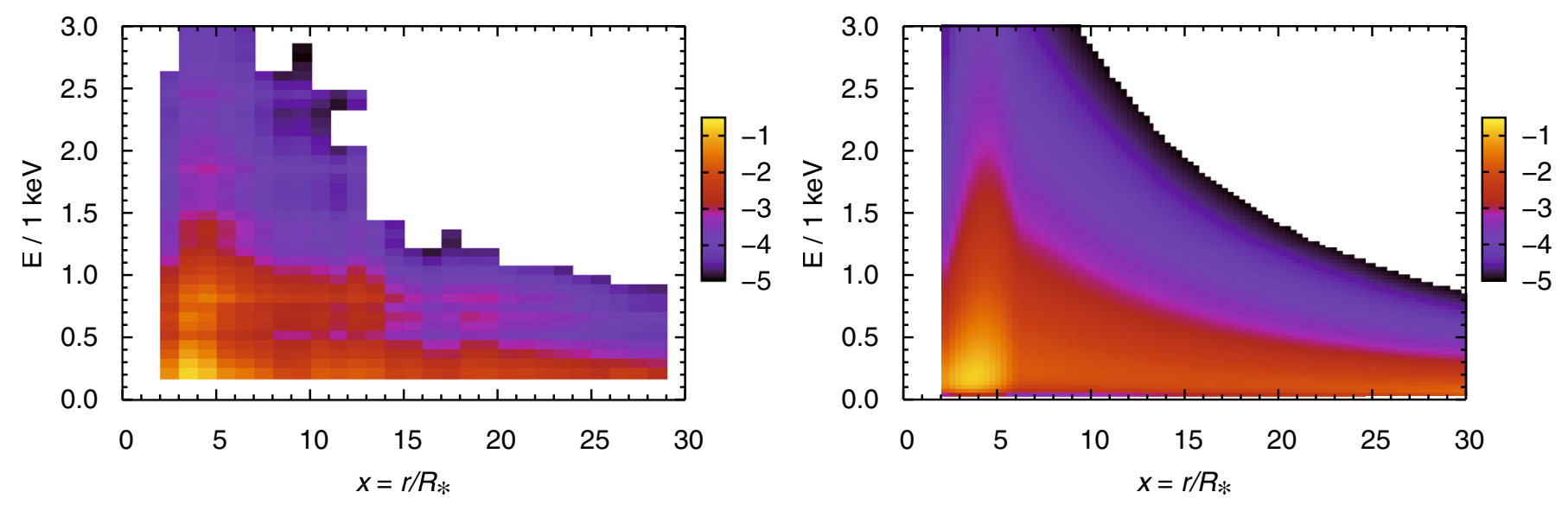

Fig. 1. Left: the frequency distribution of X-rays expressed in terms of $\log \left(\ell_{i j} / 10^{-23} \mathrm{erg} \mathrm{s}^{-1} \mathrm{~cm}^{-3} \mathrm{keV}^{-1}\right)$ as a function of radius and frequency (see Eq. (2)). Right: fit to the X-ray emissivity expressed as $\log \ell$ (Eq. (4)).

Raymond 1988) for uniformly spaced frequencies $v_{j}$ with the temperature $T(r)$. The variables $n_{\mathrm{e}}(r), n_{\mathrm{H}}(r)$, and $T(r)$ were taken from the simulation of $\zeta$ Ori A wind (Feldmeier et al. 1997a). We calculate the quantities

$\ell_{i j}=\frac{1}{\Delta v} \frac{\bar{l}_{i j}}{m_{i}}$

where

$m_{i}=\int_{r_{i}}^{r_{i+1}} r^{2} \tilde{n}_{\mathrm{e}}(r) \tilde{n}_{\mathrm{H}}(r) \mathrm{d} r$,

$\bar{l}_{i j}$ is the time average of $l_{i j k}$, and $\Delta v$ is the frequency spacing. $\tilde{n}_{\mathrm{e}}(r)$ and $\tilde{n}_{\mathrm{H}}(r)$ are the electron and hydrogen number densities from a stationary wind model corresponding to hydrodynamical simulations. This stationary model is calculated by adopting the mean mass-loss rate from the hydrodynamical simulations (Feldmeier et al. 1997a) and using a standard beta velocity law.

To provide a simpler description of the X-ray emission, we fit the values of $\ell_{i j} / 10^{-23} \mathrm{erg} \mathrm{s}^{-1} \mathrm{~cm}^{-3} \mathrm{keV}^{-1}$ by (see Fig. 1)

$\log \ell(x, \gamma)=a(x)+b(x) \gamma+c(x) \gamma^{2}+d(x) \gamma^{3}$,

where $\gamma=\log (E / 1 \mathrm{keV})$ is the logarithm of energy $E$ expressed in $\mathrm{keV}$, and $x=r / R_{*}$. The radial variation of the polynomial coefficients is given by the expressions

$a(x)=-6.087+1.836 x_{6}-0.211 x_{6}^{2}-0.112\left(x-x_{6}\right)$,

$b(x)=-4.124+0.232 x_{6}-0.132\left(x-x_{6}\right)$,

$c(x)=0.651-0.456 x_{6}$,

$d(x)=2.457-0.369 x_{6}$,

$x_{6}= \begin{cases}x, & x<6 \\ 6, & x \geq 6 .\end{cases}$

Note that $\ell=0$ for $x<2$. The X-ray emissivity in the non-LTE model can be then approximated by

$\eta_{\mathrm{X}}(r, \gamma)=\frac{f^{*}}{4 \pi} n_{\mathrm{e}} n_{\mathrm{H}} \ell\left(r / R_{*}, \gamma\right) 10^{-23} \mathrm{erg} \mathrm{s}^{-1} \mathrm{~cm}^{-3} \mathrm{keV}^{-1}$,

where $n_{\mathrm{e}}, n_{\mathrm{H}}$ are the number densities of free electrons and hydrogen in the non-LTE model. $f^{*}$ is the scaling factor (see below, Eq. (12)), which allows one to use Eq. (6) also for stars other than $\zeta$ Ori A. In Eq. (6) we introduced the factor $1 / 4 \pi$ because the emissivity $\Lambda(v, T)$ is assumed to be that in all spatial directions.

\subsection{Temperature distribution fitting}

Instead of fitting the X-ray emissivity from simulations directly, it is also possible to fit the temperature distribution of X-ray emitting gas. This approach does not explicitly depend on a particular functional form of the X-ray emissivity $\Lambda(v, T)$, but is also not completely independent of it, as the distribution of temperatures in the numerical simulations depends on the adopted form of the cooling function.

As the amount of emitted X-rays per unit volume of gas depends on the square of the density $n^{2}$, it is not realistic to fit just the distribution of temperatures. To account for the correlation of the distribution of $n^{2}$ with temperature, we evaluate for each timestep $k$ the quantity similar to the differential emission measure (e.g., Dzifčáková et al. 2008)

$\operatorname{DEM}_{i j k}=\int_{r_{i}}^{r_{i+1}} r^{2} n_{\mathrm{e}}(r) n_{\mathrm{H}}(r) \delta_{j}(T(r)) \mathrm{d} r$,

where $\delta_{j}(T(r))$ is equal to 1 for $T_{j}-\Delta T_{j} / 2<T(r)<T_{j}+\Delta T_{j} / 2$ and 0 otherwise. Here we introduced the logarithmically spaced grid of temperatures $T_{j}$ with grid size $\Delta T_{j}$. The normalised DEM is introduced as

$\mathcal{D}_{i j}=\frac{1}{\Delta T_{j}} \frac{\overline{\mathrm{DEM}}_{i j}}{m_{i}}$

where $\overline{\mathrm{DEM}}_{i j}$ is the time average of $\mathrm{DEM}_{i j k}$.

To provide a simpler description of $\mathcal{D}_{i j}$ we approximate it by a polynomial fit as

$\log \mathcal{D}(x, t)=a(x)+b(x) t$,

where $t=\log (T / 1 \mathrm{~K})$ is the logarithm of temperature expressed in units of Kelvin, and $x=r / R_{*}$. The radial variation of the coefficients $a(x), b(x)$ is given by

$a(x)=3.383+1.509 x_{6}-0.279 x_{6}^{2}+0.416\left(x-x_{6}\right)$,

$b(x)=-2.435+0.0564 x_{6}+0.00724 x_{6}^{2}-0.0783\left(x-x_{6}\right)$.

Note that $\mathcal{D}=0$ for $x<2$. For given number densities of free electrons and hydrogen the $\mathrm{X}$-ray emissivity is

$\eta_{\mathrm{X}}(r, v)=\frac{f^{*}}{4 \pi} n_{\mathrm{e}} n_{\mathrm{H}} \int_{T_{i}}^{T_{f}} \mathcal{D}\left(r / R_{*}, \log T\right) \Lambda_{v}(T) \mathrm{d} T$,

where we set $T_{i}=10^{5} \mathrm{~K}, T_{f}=2.5 \times 10^{7} \mathrm{~K}$, and $\Lambda_{v}(T)$ is the $\mathrm{X}$-ray emissivity per unit of frequency. A comparison of $\mathcal{D}$ as derived from the simulations and our fit is given in Fig. 2. 

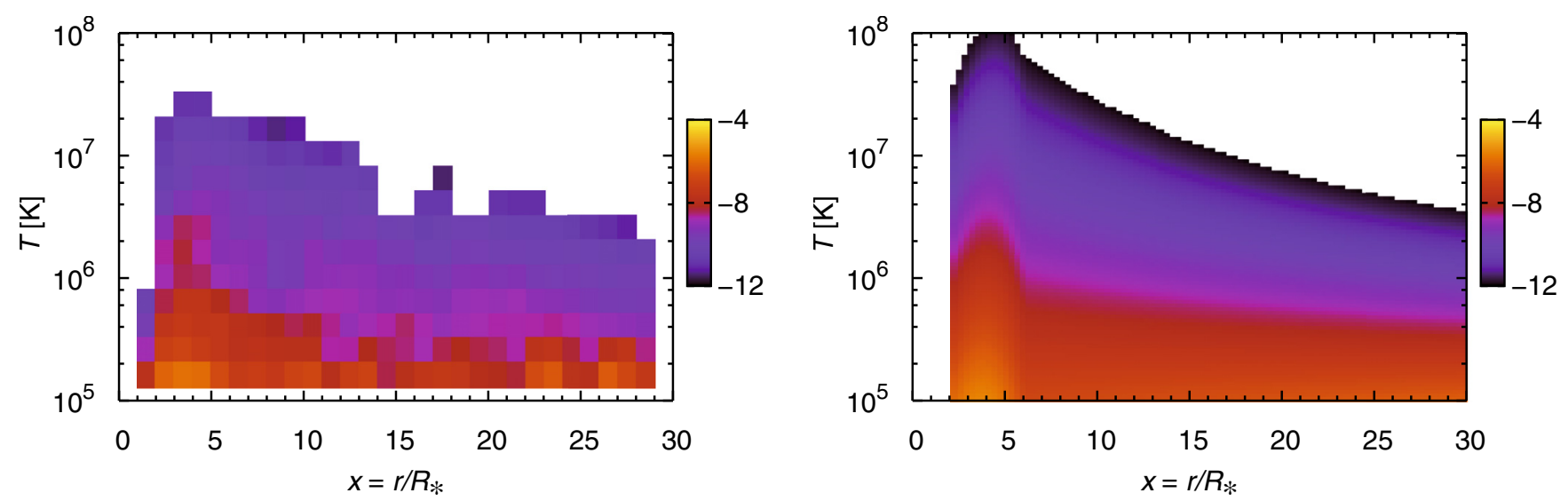

Fig. 2. Left: the temperature distribution of X-ray emitting gas expressed as $\log \mathcal{D}_{i j}$, as a function of radius. Right: fit to the temperature distribution expressed as $\log \mathcal{D}$.

We checked that both approximations of X-ray emission from hydrodynamical simulations (Eqs. (6) or (11)) and models based on exact data give similar results.

\subsection{Scaling of $X$-ray emissivity with stellar parameters}

The X-ray emission in the hydrodynamical wind simulations of Feldmeier et al. (1997a) was obtained for a mass-loss rate $\dot{M}_{\zeta \text { Ori A }}=3 \times 10^{-6} M_{\odot} /$ year, radius $R_{\zeta \text { Ori A }}=24 R_{\odot}$, and terminal velocity $v_{\zeta \text { Ori A }}=1850 \mathrm{~km} \mathrm{~s}^{-1}$ corresponding to a hydrodynamical wind model of $\zeta$ Ori A. Most of the X-rays used in these simulations originate due to the collisions of fast clouds with dense shell fragments, where the cloud formation is triggered by a turbulent photospheric seed perturbation. Consequently, it is not clear whether the derived analytical approximations are realistic also for other stars. To account for the difference between the X-ray emission in individual star wind models and $\zeta$ Ori A model of Feldmeier et al. (1997a), we introduced a scaling factor $f^{*}$ in Eqs. (6), (11).

The X-ray emissivity is given by the rate of energy dissipation by shocks. Neglecting possible dependencies on velocity and base perturbations, we expect the rate of energy dissipation to be proportional to the wind density. However, this is not what we would get from the scaling $\eta_{\mathrm{X}} \sim \rho^{2}$ in Eqs. (6), (11) with neglected dependence on the individual wind parameters, i.e. with $f^{*}=1$. The reason is that we did not take into account that the fraction of X-ray emitting material may not be the same for all stars. In the winds with similar abundances, the radiative cooling is more efficient in stars with denser winds than in stars with low-density winds. In the winds with low density, a larger fraction of the wind gas may be involved in radiating away the shock thermal energy.

Based on these arguments, we could introduce the scaling of $f^{*}$ with the density $\rho$ at a given point in the wind as $f^{*} \sim 1 / \rho$. However, this is not the most convenient scaling, as it would introduce additional dependence of $f^{*}$ on the radius, which is already accounted for in Eqs. (6), (11). Consequently, for each star the value of $f^{*}$ should be fixed. Taking into account that $\rho \sim \dot{M} /\left(r^{2} v\right)$, we introduce a representative wind density $\tilde{\rho}=$ $\dot{M} /\left(R_{*}^{2} v_{\infty}\right)$ and in our calculations use

$f^{*}=\frac{\tilde{\rho}_{\zeta \text { Ori A }}}{\tilde{\rho}}=\frac{\dot{M}_{\zeta \text { Ori A }}}{\dot{M}} \frac{R_{*}^{2}}{R_{\zeta \text { Ori A }}^{2}} \frac{v_{\infty}}{v_{\zeta \text { Ori A }}}$

where $R_{*}, \dot{M}$, and $v_{\infty}$ are radius, wind mass-loss rate, and terminal velocity of individual stars.

\section{Comparison with the "filling factor" approach}

It is informative to compare the X-ray spectrum and the temperature distribution function derived from hydrodynamic simulations in the previous section with those derived using simple "filling factor" approach. The filling factor determines the amount of X-ray emitting material.

We calculated the wind model of $\zeta$ Ori A with X-ray emission included after the "filling factor" approach of Krtička \& Kubát (2009) and derived the resulting frequency distribution of emitted X-rays and the temperature distribution of X-ray emitting gas (see Fig. 3). The adopted model assumes that the temperature of the X-ray emitting gas is given by the RankineHugoniot condition with the velocity difference related to the wind speed. Consequently, the temperature of X-ray emitting gas in Fig. 3 increases with radius and X-rays are harder in outer wind parts.

This is different from the results of hydrodynamical simulations. In the simulations the highest temperatures of X-ray emitting gas are achieved close to the star (see Fig. 2). As a result, the most energetic X-rays are emitted close to the star (see Fig. 1). Such a temperature distribution is supported by observations, as was inferred by Waldron \& Cassinelli (2007) from their extensive analysis of high-resolution X-ray spectra of $\mathrm{O}$ stars. Consequently, two important features of the X-ray emission emerge from our simulations (and from observations): the decrease of the temperature of X-ray emitting gas with radius in the outer wind, and the multitemperature distribution of X-ray emitting gas at a given point.

\section{Non-LTE models}

\subsection{Model assumptions}

As an application of derived analytical formulae, we include X-ray emission parameterised by Eqs. (11) and (12) into our stationary, spherically symmetric non-LTE wind model (see Krtička \& Kubát 2009, for a detailed description). The X-ray emission is included in the radiative transfer equation of our models. In the following we discuss just the temperature fitting approach as it seems to be more straightforward. The hydrogen and free electron densities in the X-ray source term are taken from the non-LTE models.

The excitation and ionization state of the considered elements is calculated from statistical equilibrium (non-LTE) equations. We use atomic data from the Opacity Project and the Iron 

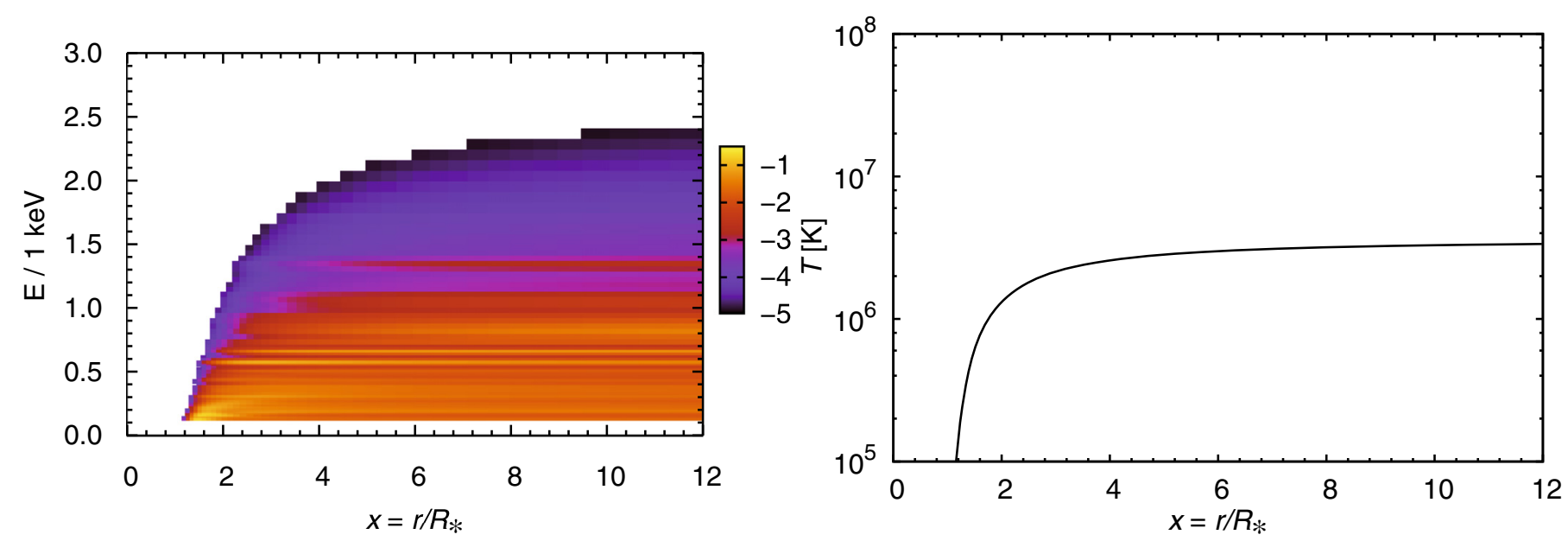

Fig. 3. Left: the frequency distribution of X-rays derived using the "filling factor" approach expressed in terms of $\log \left(\ell_{i j} / 10^{-23} \mathrm{erg} \mathrm{s}^{-1} \mathrm{~cm}^{-3} \mathrm{keV}^{-1}\right)$ as a function of radius and frequency (cf. with Fig. 1). Right: the temperature of X-ray emitting gas expressed as a function of radius (cf. with Fig. 2).

Project (Seaton 1987; Fernley et al. 1987; Luo \& Pradhan 1989; Sawey \& Berrington 1992; Seaton et al. 1992; Butler et al. 1993; Nahar \& Pradhan 1993; Hummer et al. 1993; Bautista 1996; Nahar \& Pradhan 1996; Zhang 1996; Bautista \& Pradhan 1997; Zhang \& Pradhan 1997; Chen \& Pradhan 1999). A significant part of the ionic models was taken from the TLUSTY code (Lanz \& Hubeny 2003, 2007). For phosphorus we employed data described by Pauldrach et al. (2001). Auger photoionization cross sections from individual inner-shells were taken from Verner \& Yakovlev (1995, see also Verner et al. 1993), and Auger yields were taken from Kaastra \& Mewe (1993). We use Asplund et al. (2005) solar abundance determinations in the code.

The radiative transfer equation is split in two parts, i.e., the continuum and line radiative transfer. The radiative transfer in the continuum is solved using the Feautrier method in spherical coordinates (Mihalas \& Hummer 1974; or Kubát 1993) with inclusion of all free-free and bound-free transitions of the model ions. The radiative transfer in lines is solved in the Sobolev approximation (e.g., Castor 1974) neglecting continuum opacity and line overlaps.

The radiative force is calculated in the Sobolev approximation (see Castor 1974). The corresponding line data were extracted in 2002 from the VALD database (Piskunov et al. 1995; Kupka et al. 1999). The radiative cooling and heating terms are calculated using the electron thermal balance method (Kubát et al. 1999). For the calculation of these terms we use occupation numbers derived from the statistical equilibrium equations. Finally, the continuity equation, equation of motion, and energy equation are solved iteratively to obtain the wind density, velocity, and temperature structure.

The shortest wavelength considered in the models is $4.1 \AA$ and the longest X-ray wavelength is defined as $100 \AA$.

\subsection{Test stars}

The purpose of our study is to investigate the trends in the X-ray emission known for all OB stars, rather than to study X-ray properties of individual objects. Therefore, we compiled a list of O stars that includes the objects of various luminosity classes, masses, and binarity status. By doing this, our list may be considered as representative of a diverse population of $\mathrm{O}$ stars found in real star clusters. This allows us to compare the trend in Xray luminosity of our synthetic $\mathrm{O}$ star population with the real clusters recently observed by Sana et al. (2006) and Antokhin et al. (2008).

To address the "weak wind problem" (Bouret et al. 2003; Martins et al. 2005), we also included stars that should be subject to it, i.e., their observed wind lines are much weaker than expected (the bottom four stars below the horizontal line in Table 1).

The stellar parameters (see Table 1) are taken from Lamers et al. (1995), Repolust et al. (2004), Markova et al. (2004), and Martins et al. (2005). Note that the parameters derived by Repolust et al. (2004), Markova et al. (2004), and Martins et al. (2005) were obtained using blanketed model atmospheres, i.e., they should be more reliable than the older ones. Stellar masses were obtained using evolutionary tracks (Schaller et al. 1992). The mass-loss rates in Table 1 were theoretically predicted using our models discussed in Sect. 5.1.

\section{Non-LTE models with X-ray emission from simulations}

The inclusion of X-ray emission does not significantly modify the ionization fractions close to the star below the critical point radius where the mass-loss rate is fixed. Consequently, X-rays do not significantly modify the wind mass-loss rate, but may modify the wind terminal velocity by a few percent (Krtička \& Kubát 2009).

\subsection{X-ray luminosity}

A comparison of the predicted X-ray luminosities for individual stars and the mean observational trends is given in Fig. 4. The predicted X-ray luminosities for stars with optically thick winds $\left(L \gtrsim 10^{5} L_{\odot}\right)$ are on average lower roughly by a factor of three than the observed ones. This corresponds to the results of Feldmeier et al. (1997a).

The derived slope of the $L_{X}-L$ relation for stars with optically thick winds, $L_{X} \sim L^{1.0}$, is in better agreement with observations. The cause of the decrease of the slope (compared to results obtained for a fixed filling factor as a free parameter, Krtička \& Kubát 2009) can be most easily understood from Fig. 1, which shows that $\ell(x, \gamma)$ decreases with $x$ (i.e., with radius). Consequently, the filling factor also decreases with radius, supporting the explanation of Owocki \& Cohen (1999). Also the 
Table 1. Stellar parameters of studied O stars.

\begin{tabular}{rccccccc}
\hline \hline Star & HD & $\begin{array}{c}\text { Sp. } \\
\text { type }\end{array}$ & $\begin{array}{c}R_{*} \\
{\left[R_{\odot}\right]}\end{array}$ & $\begin{array}{c}M \\
{\left[M_{\odot}\right]}\end{array}$ & $\begin{array}{c}T_{\text {eff }} \\
{\left[10^{3} \mathrm{~K}\right]}\end{array}$ & $\begin{array}{c}L \\
{\left[10^{5} L_{\odot}\right]}\end{array}$ & $\begin{array}{c}\dot{M} \\
{\left[M_{\odot} / \text { year }\right]}\end{array}$ \\
\hline$\xi$ Per & 24912 & O7.5IIIe & 14.0 & 36 & 35.0 & 2.64 & $4.6 \times 10^{-7}$ \\
$\alpha$ Cam & 30614 & O9.5Iae & 27.6 & 43 & 30.9 & 6.23 & $1.5 \times 10^{-6}$ \\
$\lambda$ Ori A & 36861 & O8 III & 12.3 & 30 & 36.0 & 2.28 & $5.1 \times 10^{-7}$ \\
$\iota$ Ori A & 37043 & O9III & 21.6 & 41 & 31.4 & 4.07 & $6.3 \times 10^{-7}$ \\
$\zeta$ Ori A & 37742 & O9Iab & 24.0 & 34 & 31.5 & 5.09 & $1.5 \times 10^{-6}$ \\
15 Mon & 47839 & O7Ve & 9.9 & 32 & 37.5 & 1.74 & $2.8 \times 10^{-7}$ \\
& 54662 & O7III & 11.9 & 38 & 38.6 & 2.82 & $8.9 \times 10^{-7}$ \\
& 93204 & O5V & 11.9 & 41 & 40.0 & 3.25 & $1.4 \times 10^{-6}$ \\
$\zeta$ Oph & 149757 & O9V & 8.9 & 21 & 32.0 & 0.75 & $4.6 \times 10^{-8}$ \\
63 Oph & 162978 & O8III & 16.0 & 40 & 37.1 & 4.35 & $2.0 \times 10^{-6}$ \\
68 Cyg & 203064 & O8e & 15.7 & 38 & 34.5 & 3.13 & $6.1 \times 10^{-7}$ \\
19 Cep & 209975 & O9Ib & 22.9 & 47 & 32.0 & 4.93 & $8.6 \times 10^{-7}$ \\
$\lambda$ Cep & 210839 & O6Iab & 19.6 & 51 & 38.2 & 7.34 & $6.4 \times 10^{-6}$ \\
\hline AE Aur & 34078 & O9.5Ve & 7.5 & 20 & 33.0 & 0.60 & $1.4 \times 10^{-8}$ \\
$\mu$ Col & 38666 & O9.5V & 6.6 & 19 & 33.0 & 0.46 & $7.8 \times 10^{-9}$ \\
& 42088 & O6.5V & 9.6 & 31 & 38.0 & 1.72 & $3.4 \times 10^{-7}$ \\
& 46202 & O9V & 8.4 & 21 & 33.0 & 0.75 & $2.3 \times 10^{-8}$ \\
\hline
\end{tabular}

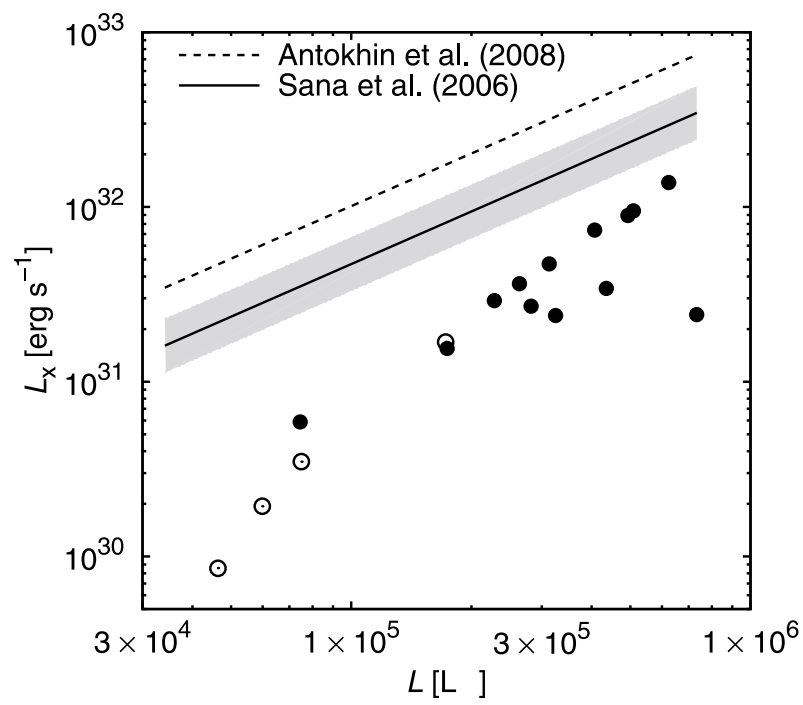

Fig. 4. The dependence of the total X-ray luminosity on the bolometric luminosity calculated using non-LTE models with X-ray emissivity after Eqs. (11) and (12) (filled and empty circles) for individual stars. This is compared with the mean observational relations (corrected for the interstellar absorption) derived by Antokhin et al. (2008) and Sana et al. (2006, with uncertainty). Empty circles denote values for stars showing the "weak wind problem".

dependence of $f^{*}$ on the stellar parameters contributes to the decrease of the slope of the $L_{X}-L$ relation, but the contribution of this parameter is sensitive to the stellar sample considered.

For stars with optically thin wind $\left(L \lesssim 10^{5} L_{\odot}\right)$, even the improved inclusion of $\mathrm{X}$-ray emission is not capable of explaining the observed slope of the $L_{X}-L$ relation. As indicated above, the dependence of the predicted X-ray luminosity of these stars on stellar luminosity is steeper than for stars with high luminosity. However, this is not supported by observations. The $L_{X}-L$ relation of low luminosity stars is either the same as that of high luminosity stars, or, for B stars, the slope is even less steep (Sana et al. 2006). The reason for this discrepancy between observation and theory is unclear. Note that for these stars the shock cooling length could be comparable with the hydrodynamical

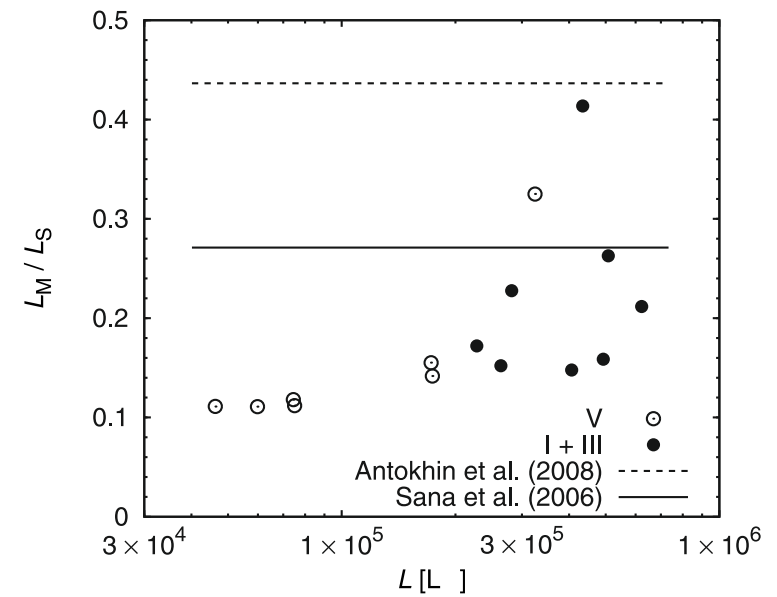

Fig. 5. Comparison between the predicted ratio of X-ray luminosities in the medium and soft energy bands for individual stars and the mean trend derived from observations. Main sequence stars are denoted with empty symbols, giants and supergiants with filled symbols.

scale (Cohen et al. 2008; Krtička \& Kubát 2009). The fact that a substantial fraction of the wind is too hot to give a significant signature in the ultraviolet spectrum of the star could be an explanation for the weak-wind problem (Krtička \& Kubát 2009).

\subsection{The energy distribution of $X$-rays}

Sana et al. (2006) and Antokhin et al. (2008) divide the $\mathrm{X}$-rays into three energy bands, soft $(0.5-1.0 \mathrm{keV})$, medium $(1.0-2.5 \mathrm{keV})$, and hard $(2.5-10.0 \mathrm{keV})$. The observations show that the slope of the $L_{X}-L$ relation is roughly the same in the soft and medium band as in the total X-ray emission, whereas the observations in the hard band show a large dispersion.

Our calculations show a somewhat different result, as can be seen from Fig. 5, where we plot the ratio of the X-ray luminosities in the medium $\left(L_{\mathrm{M}}\right)$ and soft $\left(L_{\mathrm{S}}\right)$ energy bands. The predicted slope of the $L_{X}-L$ relation in the medium band is steeper than that in the soft band, consequently the ratio of $L_{\mathrm{M}} / L_{\mathrm{S}}$ increases with luminosity. The reason for this behaviour is the inverse proportionality of X-ray opacity and energy 

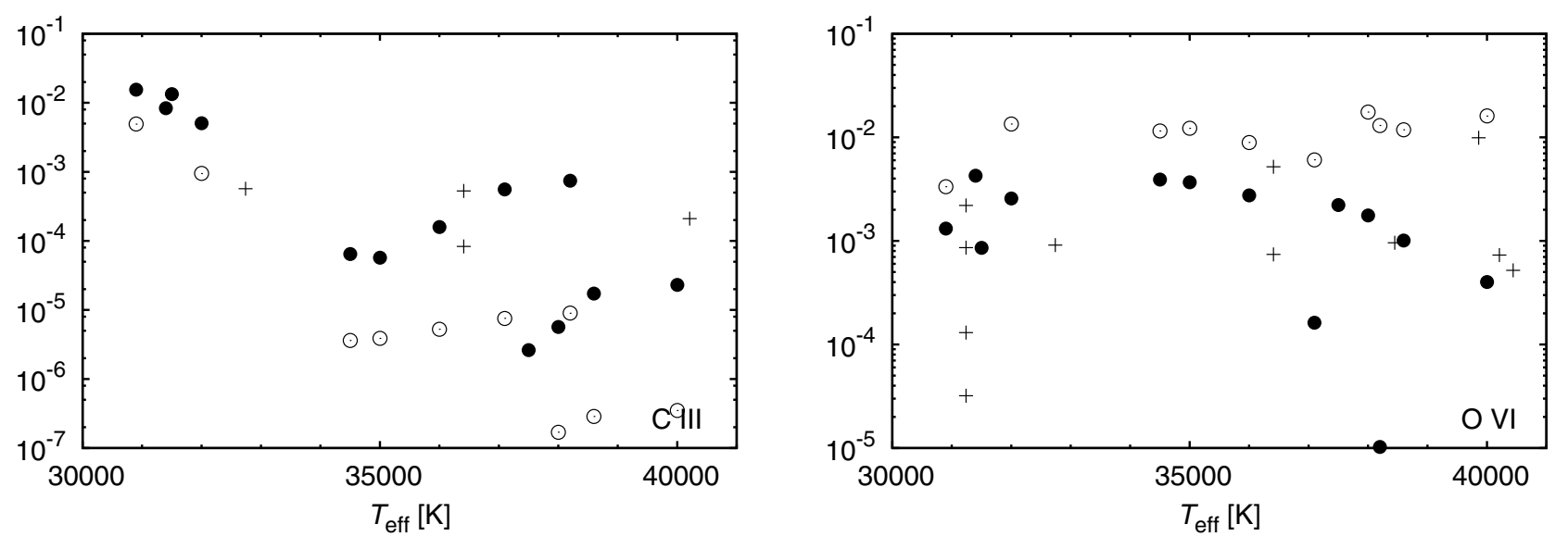

Fig. 6. Ionization fractions as a function of the effective temperature for individual stars from our sample (only stars with $\dot{M}>10^{-7} M_{\odot} /$ year are included here) at the point where the radial velocity $v=0.5 v_{\infty}$. Filled circles $\bigcirc$ refer to the present models with X-ray emission from hydrodynamical simulations, open circles $\odot$ denote values taken from former non-LTE models with X-ray emission described using filling factor (Krtička \& Kubát 2009). The ionization fractions derived from observations were adopted from Massa et al. (2003, for LMC stars, plus signs +).

(e.g. Oskinova et al. 2006; Krtička \& Kubát 2009). For stars with low luminosities, the wind density is low, and most of the emitted X-rays will be emergent. On the other hand, for stars with high luminosities, density and opacity values become large, more flux is absorbed in the soft than in the medium energy band, consequently the emergent $\mathrm{X}$-rays become harder. This also explains why the predicted hardness of $\mathrm{X}$-rays is greater for giants and supergiants than for main sequence stars (see Fig. 5). Finally, although from our models it follows that the predicted dispersion of X-ray luminosities is indeed higher in the hard band than in the medium and soft energy bands, there is still a clearly discernible $L_{\mathrm{X}}-L$ relation even in the hard band.

\subsection{Ionization fractions}

The presence of X-rays may also influence the ionization fractions of highly ionised ions. We plot in Fig. 6 the predicted ionization fractions in comparison with those derived from observation as a function of effective stellar temperature. Note that here we use the ionization fractions derived for LMC stars (Massa et al. 2003), because their observational sample includes a large number of ionization stages, the fractions of which are given as a function of velocity, and the wind parameters of LMC stars are not significantly different from Galactic ones. Since several parameters except the effective temperature (e.g., the wind density) may influence the ionization fractions, the graphs in Fig. 6 are not monotonic.

In Fig. 6 we also plot the results of Krtička \& Kubát (2009), which were obtained using a simpler inclusion of X-rays assuming a constant filling factor (see also Sect. 4). Still, the emergent $\mathrm{X}$-ray luminosities from these models roughly correspond to the observed ones. Rather surprisingly, although the non-LTE models calculated with X-ray emission from hydrodynamical wind simulations give a too low emergent X-ray luminosity, the ionization structure of these models corresponds in general much better to the trends derived from observations.

This better agreement between theoretical and observational ionization fractions may be due to an overall lower X-ray emissivity. To test this, we calculated models with a simplified treatment of X-ray emission after Krtička \& Kubát (2009), but assuming the same X-ray luminosity as that predicted by hydrodynamical simulations. Our results show that even in this case the use of X-ray emissivity based on hydrodynamical simulation gives better agreement with observations. We conclude that the hydrodynamical simulations give a more realistic dependence of the X-ray emissivity on frequency.

\section{Discussion}

The remaining discrepancy between theory and observation may originate in our neglect of macroclumping (sometimes also termed porosity). The macroclumping may cause a lower opacity in the X-ray domain, which leads to a higher predicted X-ray luminosity. From Fig. 17 in Oskinova et al. (2004), it follows that an increase of X-ray luminosity by a factor of 3 would be easily achievable. Accounting for macroclumping reduces the wavelength dependence of opacity. In its limit (when clumps are fully opaque), the opacity becomes grey (Oskinova et al. 2004).

Wind inhomogeneities should also affect the ionization fractions. Optically thin inhomogeneities (often referred to as "microclumping") lead generally to lower ionization stages (de Koter et al. 2008; Krtička et al. 2008), hence clumping with increased X-ray emissivity could be another way to explain the X-ray luminosities and ionization fractions derived from observation.

Our hydrodynamical wind simulations assume 1D spherical symmetry, thus all wind structures correspond to full spherical shells. Therefore, we have at present a very limited knowledge of real wind clumping.

To be consistent with the hydrodynamical simulations, we use the Raymond-Smith X-ray spectral code (Raymond \& Smith 1977; Raymond 1988), although it would be possible to use codes based on up-to-date atomic data. Our test using the APEC subroutine available in XSPEC (Arnaud 1996; Smith et al. 2001) showed that there is a relatively good agreement between the fits using this and the Raymond-Smith code Eq. (5).

\section{Summary}

We provide analytical approximations of the X-ray emission predicted by hydrodynamical simulations of hot star winds by Feldmeier et al. (1997a). The X-ray emission derived from the hydrodynamical simulations has two distinctive aspects. First, the temperature of X-ray emitting gas decreases with radius in the outer wind. Second, the temperature of the X-ray emitting 
gas is described by a distribution function, which is more realistic than assuming just one temperature at a given point.

We include these approximations in non-LTE models (Krtička \& Kubát 2009) and for selected stars compare the resulting X-ray luminosities, energy distribution of emergent $\mathrm{X}$-rays, and ionization structure with observational results. We conclude that although the predicted X-ray luminosity is slightly underestimated, the resulting ionization structure is in relatively good agreement with observations. Earlier papers debated whether the theory of line-driven winds can explain the observed $L_{\mathrm{X}} \sim L$ relation. Our results reproduce this scaling.

Acknowledgements. We thank the referee for his/her comments that helped to improve the manuscript. This work was supported by grant GA ČR 205/08/0003. L.M.O. acknowledges the support of DLR grant 50 OR 0804.

\section{References}

Antokhin, I. I., Rauw, G., Vreux, J.-M., van der Hucht, K. A., \& Brown, J. C. 2008, A\&A, 477, 593

Arnaud, K. A. 1996, Astronomical Data Analysis Software and Systems V, ed. G. Jacoby, \& J. Barnes (San Francisco: ASP), 17

Asplund, M., Grevesse, N., \& Sauval, A. J. 2005, Cosmic Abundances as Records of Stellar Evolution and Nucleosynthesis, ed. T. G. Barnes III, \& F. N. Bash (San Francisco: ASP), ASP Conf. Ser., 336, 25

Babel, J., \& Montmerle, T. 1997, A\&A, 323, 121

Benaglia, P., Vink, J. S., Martí, J., et al. 2007, A\&A, 467, 1265

Bautista, M. A. 1996, A\&AS, 119, 105

Bautista, M. A., \& Pradhan, A. K. 1997, A\&AS, 126, 365

Berghöfer, T. W., Schmitt, J. H. M. M., Danner, R., \& Cassinelli, J. P. 1997, A\&A, 322, 167

Bianchi, L., Bohlin, R., \& Massey, P. 2004, ApJ, 601, 228

Bouret, J.-C., Lanz, T., Hillier, D. J., et al. 2003, ApJ, 595, 1182

Burgers, J. M. 1974, The Nonlinear Diffusion Equation (Dordrecht: Reidel)

Butler, K., Mendoza, C., \& Zeippen, C. J. 1993, J. Phys. B., 26, 4409

Castor, J. I. 1974, MNRAS, 169, 279

Chen, G. X., \& Pradhan, A. K. 1999, A\&AS, 136, 395

Cooke, B. A., Fabian, A. C., \& Pringle, J. E. 1978, Nature, 273, 645

Chlebowski, T., Harnden, F. R., \& Sciortino, S. 1989, ApJ, 341, 427

Cohen, D. H., Kuhn, M. A., Gagné, M., Jensen, E. L. N., \& Miller, N. A. 2008, MNRAS, 386, 1855

de Koter, A., Vink, J. S., \& Muijres, L. 2008, in Clumping in Hot Star Winds, ed. W.-R. Hamann, A. Feldmeier, \& L. Oskinova, 47

Dzifčáková, E., Kulinová, A., Chifor, C., et al. 2008, A\&A, 488, 311

Feldmeier, A. 1995, A\&A, 299, 523

Feldmeier, A., Puls, J., \& Pauldrach, A. W. A. 1997a, A\&A, 322, 878

Feldmeier, A., Kudritzki, R.-P., Palsa, R., Pauldrach, A. W. A., \& Puls, J. 1997b, A\&A, 320, 899

Feldmeier, A., Oskinova, L., \& Hamann, W.-R. 2003, A\&A, 403, 217

Fernley, J. A., Taylor, K. T., \& Seaton, M. J. 1987, J. Phys. B, 20, 6457

Hedstrom, G. W. 1979, J. Comp. Phys., 30, 222

Hillier, D. J., Kudritzki, R. P., Pauldrach, A., et al. 1993, A\&A, 276

Hummer, D. G., Berrington, K. A., Eissner, W., et al. 1993, A\&A, 279, 298

Kaastra, J. S., \& Mewe, R. 1993, A\&AS, 97, 443

Krtička, J. 2006, MNRAS, 367, 1282

Krtička, J., \& Kubát, J. 2009, MNRAS, 394, 2065

Krtička, J., Puls, J., \& Kubát, J. 2008, in Clumping in Hot Star Winds, ed. W.-R. Hamann, A. Feldmeier, \& L. Oskinova, 111

Kubát, J. 1993, Ph.D. Thesis, Astronomický ústav AV ČR, Ondřejov

Kubát, J., Puls, J., \& Pauldrach, A. W. A. 1999, A\&A, 341, 587

Kudritzki, R. P., \& Puls, J. 2000, ARA\&A, 38, 613
Kupka, F., Piskunov, N. E., Ryabchikova, T. A., Stempels, H. C., \& Weiss, W. W. 1999, A\&AS, 138, 119

Lamb, H. 1945, Hydrodynamics (New York: Dover)

Lamers, H. J. G. L. M., Snow, T. P., \& Lindholm, D. M. 1995, ApJ, 455, 269

Langer, S. H., Chanmugam, G., \& Shaviv, G. 1981, ApJ, 245, L23

Lanz, T., \& Hubeny, I. 2003, ApJS, 146, 417

Lanz, T., \& Hubeny, I. 2007, ApJS, 169, 83

Lucy, L. B. 1984, ApJ, 284, 351

Lucy, L. B., \& Solomon, P. M. 1970, ApJ, 159, 879

Luo, D., \& Pradhan, A. K. 1989, J. Phys. B, 22, 3377

MacGregor, K. B., Hartmann, L., \& Raymond, J. C. 1979, ApJ, 231, 514

Markova, N., Puls, J., Repolust, T., \& Markov, H. 2004, A\&A, 413, 693

Martins, F., Schaerer, D., Hillier, D. J., et al. 2005, A\&A, 441, 735

Mihalas, D., \& Hummer, D. G. 1974, ApJS, 28, 343

Massa, D., Fullerton, A. W., Sonneborn, G., \& Hutchings, J. B. 2003, ApJ, 586, 996

MacFarlane, J. J., Cohen, D. H., \& Wang, P. 1994, ApJ, 437, 351

Nahar, S. N., \& Pradhan, A. K. 1993, J. Phys. B, 26, 1109

Nahar, S. N., \& Pradhan, A. K. 1996, A\&AS, 119, 509

Oskinova, L. M., Feldmeier, A., \& Hamann, W.-R. 2004, A\&A, 422, 675

Oskinova, L. M., Feldmeier, A., \& Hamann, W.-R. 2006, MNRAS, 372, 313

Owocki, S. P. 1991, in Stellar Atmospheres: Beyond Classical Models, ed. L.

Crivellari, I. Hubeny, \& D. G. Hummer (Dordrecht: Kluwer), 235

Owocki, S. P., \& Cohen, D. H. 1999, ApJ, 520, 833

Owocki, S. P., \& Puls, J. 1996, ApJ, 462, 894

Owocki, S. P., \& Rybicki, G. B. 1985, ApJ, 299, 265

Owocki, S. P., Castor, J. I., \& Rybicki, G. B. 1988, ApJ, 335, 914

Owocki, S. P., Gayley, K. G., \& Shaviv, N. J. 2004, ApJ, 616, 525

Pauldrach, A., Puls, J., \& Kudritzki, R. P. 1986, A\&A, 164, 86

Pauldrach, A. W. A., Hoffmann, T. L., \& Lennon, M. 2001, A\&A, 375, 161

Piskunov, N. E., Kupka, F., Ryabchikova, T. A., Weiss, W. W., \& Jeffery, C. S. 1995, A\&AS, 112, 525

Pittard, J. M. 2009, MNRAS, 396, 1743

Poe, C. H., Owocki, S. P., \& Castor, J. I. 1990, ApJ, 358, 199

Prilutskii, O. F., \& Usov, V. V. 1976, AZh, 53, 6

Puls, J., Markova, N., Scuderi, S., et al. 2006, A\&A, 454, 625

Puls, J., Markova, N., \& Scuderi, S. 2008a, Mass Loss from Stars and the Evolution of Stellar Clusters, ed. A. de Koter, L. Smith, \& R. Waters (San Francisco: ASP), 101

Puls, J., Vink, J. S., \& Najarro, F. 2008b, A\&ARv, 16, 209

Raymond, J. C. 1988, in Hot Thin Plasmas in Astrophysics, NATO ASI Series C, ed. R. Pallavicini, 249, 3

Raymond, J. C., \& Smith, B. W. 1977, ApJS, 35, 419

Repolust, T., Puls, J., \& Herrero, A. 2004, A\&A, 415, 349

Risken, H. 1996, The Fokker-Planck Equation (Heidelberg: Springer)

Sana, H., Rauw, G., Nazé, Y., Gosset, E., \& Vreux, J.-M. 2006, MNRAS, 372, 661

Sawey, P. M. J., \& Berrington, K. A. 1992, J. Phys. B, 25, 1451

Schaller, G., Schaerer, D., Meynet, G., \& Maeder, A. 1992, A\&AS, 96, 269

Seaton, M. J. 1987, J. Phys. B, 20, 6363

Seaton, M. J., Zeippen, C. J., Tully, J. A., et al. 1992, Rev. Mex. Astron. Astrofis., 23, 19

Smith, R. K., Brickhouse, N. S., Liedahl, D. A., \& Raymond, J. C. 2001, ApJ, 556, 91

Thompson, K. W. 1987, J. Comp. Phys., 68, 1

Thompson, K. W. 1990, J. Comp. Phys., 89, 439

ud-Doula, A., \& Owocki, S. P. 2002, ApJ, 576, 413

van Leer, B. 1977, J. Comp. Phys., 23, 276

Verner, D. A., \& Yakovlev, D. G. 1995, A\&AS, 109, 125

Verner, D. A., Yakovlev, D. G., Band, I. M., \& Trzhaskovskaya, M. B. 1993, Atomic Data and Nuclear Data Tables, 55, 233

Vink, J. S., de Koter, A., \& Lamers, H. J. G. L. M. 2000, A\&A, 362, 295

Vink, J. S., de Koter, A., \& Lamers, H. J. G. L. M. 2001, A\&A, 369, 574

Waldron, W. L., \& Cassinelli, J. P. 2007, ApJ, 668, 456

Zhang, H. L. 1996, A\&AS, 119, 523

Zhang, H. L., \& Pradhan, A. K. 1997, A\&AS, 126, 373 